\title{
Prevalência e susceptibilidade de leveduras vaginais
}

\author{
Prevalence and susceptibility of vaginal yeast
}

Leonilda Chiari Galle'; Maria José Soares Mendes Gianinni²

\begin{abstract}
unitermos resumo
Candidiase

Fluido vaginal

Antifungigrama

Entre as vulvovaginites, a candidíase é apontada como a causa mais freqüente em mulheres na idade fértil. Atualmente, várias pesquisas mostram aumento na freqüência das espécies não-albicans e grande preocupação com episódios de repetição, assim como sua relação com a resistência ao tratamento. Objetivo: O presente estudo teve como objetivo verificar a distribuição de gêneros e espécies de leveduras causadoras de vaginite e analisar o perfil de sensibilidade das leveduras frente às drogas antifúngicas. Material e método: Foram colhidas amostras de fluido vaginal de 250 pacientes para cultura, realizados identificação e antifungigrama dos isolados. Resultados: Leveduras do gênero Candida estavam presentes em $27,6 \%$ das amostras. Candida albicans foi a levedura mais isolada em $74 \%$ dos casos, seguida de Candida glabrata, em 14,5\%; Candida tropicalis, em 7,3\%; e Candida parapsilosis, em 4,3\%. Todos os isolados Candida albicans foram sensíveis à anfotericina $\mathrm{B}$, e apenas um isolado da espécie não-albicans apresentou concentração inibitória mínima (CIM) mais elevada $(2 \mu \mathrm{g} / \mathrm{ml})$. Em Candida albicans, 5,9\% das amostras mostraram-se sensíveis, dependendo da dose de fluconazol, e 9,8\%, resistentes. Apenas um isolado mostrou-se resistente, com CIM de $8 \mu \mathrm{g} / \mathrm{ml}$, para itraconazol. Nas espécies não-albicans, $11,7 \%$ dos isolados foram considerados resistentes ao fluconazol e 23,5, ao itraconazol. Conclusão: Candida albicans foi a espécie mais freqüentemente encontrada na microbiota vaginal; no entanto, outras espécies foram também comuns nessa população. Porcentual importante de isolados de Candida albicans e não-albicans foi resistente a fluconazol e itraconazol, mostrando a importância de realização de testes de identificação e antifungigrama para os episódios de candidíase vaginal.
\end{abstract}

\section{abstract}

Among the vulvovaginitis, candidiasis is the most frequent cause in women at reprodutive age of and several researches have shownd an increase in the frequency of the species non-albicans, and a great concern with repetitive episodes, as well as its relationship with the resistance to the treatment. Objective: The purpose of this study was to verify the yeast species that cause vaginitis and their susceptibility to antifungal agents. Material and Method: Vaginal swabs were colleted from 250 patients and were evaluated for culture, species identification and susceptibility to antifungal agents. Results: Candida was present in $27.6 \%$ of the samples. Candida albicans was the predominant species (74\%), followed by Candida glabrata (14.5\%), Candida tropicalis (7,3\%) and Candida parapsilosis (4,3\%). All the C. albicans isolates were susceptible to anfotericin B and only one sample of non-albicans showed greater MIC $(2 \mu \mathrm{g} / \mathrm{mL}) .5 .9 \%$ of C. albicans strains were susceptible-dose dependent to FLU and 9,8\% resistant. Only one sample showed resistance to itraconazol, with CIM 8 $8 \mathrm{~g} / \mathrm{mL}$. In the species non-albicans, $11.8 \%$ of the isolates were considered resistant to fluconazol and $23.5 \%$ to itraconazol. Conclusion: Candida albicans is the most frequent in the vaginal microenviroment, however non-albicans are common vaginal isolates even in a primary care population. The species isolated are less susceptible to fluconazole and itraconazole than most C. albicans, showing the importance of the performance of the identification tests and susceptibility to antifungal agents of vulvovaginal candidiasis. key words

Candidiasis

Vaginal fluid

Antifungal agents

susceptibility

1. Mestre em Análises Clínicas; professora de Citologia Clínica da Faculdade de Farmácia da Universidade do Oeste Paulista (UNOESTE); biologista do Instituto Adolfo Lutz/Laboratório de Presidente Prudente.

2. Professora-adjunta do Departamento de Análises Clínicas da Faculdade de Ciências Farmacêuticas, Universidade Estadual Paulista (UNESP)/campus de Araraquara.

Tese apresentada ao programa de Pós-Graduação em Análises Clínicas da Faculdade de Ciências Farmacêuticas da UNESP/campus de Araraquara para obtenção do Título de Mestre em Análises Clínicas no ano de 2002.

Esta pesquisa foi realizada no Instituto Adolfo Lutz/Laboratório Regional de Presidente Prudente e no Laboratório de Micologia da Faculdade de Ciências Farmacêuticas da UNESP/campus de Araraquara. 


\section{Introdução}

A candidíase vulvovaginal (CVV) é a segunda causa mais freqüente de vulvovaginite. Estima-se que $75 \%$ das mulheres terão um episódio de CVV ao longo de suas vidas, sendo que $40 \%$ a $50 \%$ destas teriam a segunda infecção e cerca de 5\% poderiam adquirir padrão crônico, com episódios de repetição ${ }^{(6,16,17)}$.

Candida spp é responsável por $15 \%$ a $25 \%$ dos casos de vaginites, sendo causada por isolados que habitam as mucosas vaginal e digestiva e que crescem em meio favorável ao seu desenvolvimento ${ }^{(3,17)}$. Candida albicans está presente como microbiota vaginal normal em $15 \%$ a $20 \%$ das mulheres adultas saudáveis e em $30 \%$ a $40 \%$ das grávidas ${ }^{(5,6,19)}$. Outras espécies como Candida glabrata e Candida parapsilosis podem estar presentes em proporções relativamente menores ${ }^{(2)}$

Nos Estados Unidos, a incidência de vaginite micótica dobrou entre 1989 e 1990, coincidindo com aumento de $80 \%$ no uso de antimicóticos no mesmo período ${ }^{(7)}$. O diagnóstico da CVV deve ser bem estabelecido, com todos os critérios clínicos e exames laboratoriais adequados, para que não ocorram erros e o uso desnecessário de drogas antimicóticas. $\mathrm{O}$ autodiagnóstico da CVV, sem exame microscópico e/ou avaliação clínica, dificulta os estudos epidemiológicos e pode induzir resistência do microrganismo ao tratamento convencional.

De modo geral, os estudos demonstram através da cultura do fluido vaginal que Candida albicans está presente em $84 \%$ dos casos de CVV, seguida de Candida glabrata $(11,7 \%)$, Candida tropicalis (5,3\%), Candida krusei $(2,6 \%)$ e outras espécies $(2 \%)^{(8)}$. Em nosso meio ${ }^{(10)}$, foram observadas prevalências de $86,4 \%$ para C. albicans, 4,5\% para $C$. glabrata, 3,9\% para C. parapsilosis, 2,7\% para C. tropicalis e 1,4\% para outras espécies.

O presente estudo tem como objetivo verificar a distribuição de espécies de leveduras causadoras de vaginite e analisar o perfil de sensibilidade das leveduras frente às drogas antifúngicas.

\section{Material e método}

Independente de sintomas, foi colhido fluido vaginal de 250 pacientes para a realização de pesquisa direta e isolamento de leveduras através de cultura, no período entre outubro de 2000 e setembro de 2001.

Para pesquisa direta, o esfregaço do fluido vaginal de cada paciente foi corado pelo método de Gram e examina- do microscopicamente. Os resultados foram considerados positivos na presença de hifas e/ou blastoconídios. Para isolamento de leveduras, as amostras foram distribuídas em tubos com ágar-Sabouraud e cloranfenicol e incubadas à $25^{\circ} \mathrm{C}$ por até 15 dias. As culturas que não apresentaram qualquer crescimento foram consideradas negativas.

Nos casos em que a cultura foi positiva, as colônias foram submetidas a exame microscópico, com solução salina, para confirmação do crescimento de leveduras. Todas aquelas com características macroscópicas diversas foram plaqueadas em meio Chromagar Candida, para isolamento de múltiplas espécies. As colônias com morfologia uniforme foram reisoladas em placas para obtenção de colônias puras e, posteriormente, submetidas às provas para identificação de gênero e espécie, segundo Lacaz et al.(9). As provas foram realizadas com uma cepa controle positivo Candida albicans ATCC 64548.

As espécies consideradas não-C. albicans foram identificadas através de sistema de identificação denominado API-20 C AUX (Bio Meurieux, França).

O uso da técnica de microdiluição permitiu determinar a sensibilidade in vitro de todas as leveduras isoladas aos antifúngicos de uso convencional: fluconazol (Pfizer Laboratórios do Brasil), itraconazol (Janssen-Cilag do Brasil) e anfotericina B (Sigma, Illinois, EUA) utilizando metodologia M-27-A, NCCLS ${ }^{(11)}$ e modificada por Rodriguez-Tudella ${ }^{(14)}$.

As drogas foram conservadas ao abrigo da luz até o momento do uso. Para realização das provas, solução concentrada foi preparada e, com cada solução-mãe, foram feitas dez concentrações decrescentes de antifúngicos, que variaram entre $16 \mu \mathrm{g} / \mathrm{ml} \mathrm{e} 0,03 \mu \mathrm{g} / \mathrm{ml}$ para anfotericina B e itraconazol e, de $64 \mu \mathrm{g} / \mathrm{ml}$ a $0,12 \mu \mathrm{g} / \mathrm{ml}$ para fluconazol.

A concentração inibitória mínima foi determinada utilizando microplacas de fundo plano, com 96 cavidades distribuídas em oito fileiras de $\mathrm{A}$ até $\mathrm{H}$, com 12 cavidades cada uma. Nas colunas de números 2 a 11, foram dispensados $100 \mu$ lde cada solução de antifúngico, da maior até a menor concentração. Reservou-se a coluna número 12 para controle positivo de crescimento. Em seguida foram dispensados $200 \mu \mathrm{l}$ do meio nas cavidades da coluna 1, como controle de esterilidade.

O meio de cultura utilizado foi o RPMI 1640 (Sigma, Illinois, EUA) tamponado com ácido morfopropileno sulfônico (MOPS) 1,65M, pH 7,2, acrescido de L-glutamina $200 \mathrm{mM}$ e $2 \%$ de glicose para otimizar o crescimento do fungo.

Os inóculos foram preparados ressuspendendo, em solução salina esterilizada, colônias com crescimento de 
24 horas, em meio de ágar-Sabouraud sem cloranfenicol, feitas de cada uma das amostras. A turbidez foi ajustada para corresponder a $5 \times 10^{6} \mathrm{UFC} / \mathrm{ml}$ e, após homogeneização, dispensaram-se 100ul da solução nas últimas 11 cavidades de cada fileira. Para controle de qualidade em todas as placas, realizou-se também o ensaio com a cepa-padrão Candida parapsilosis (ATCC 22019). As placas foram devidamente fechadas e, no caso de anfotericina $B$, embaladas com papel alumínio, para incubação a $37^{\circ} \mathrm{C}$ por 24 horas, sob agitação.

A determinação da concentração inibitória mínima (CIM) foi feita, após a leitura a $405 \mathrm{~nm}$ automatizada, por leitor de microplacas (ELISA), da densidade ótica de cada orifício. $\mathrm{O}$ aparelho foi programado de forma que considerou a coluna 1 como branco dos reagentes. Considerou-se como controle positivo, com $100 \%$ de crescimento, o valor da densidade ótica obtida no orifício número 12.

A CIM para cada droga foi determinada com $80 \%$ e $50 \%$ de inibição do crescimento. A CIM 80\% foi determinada como a menor concentração da droga, com valor de absorbância menor, ou igual, aos $20 \%$ do valor lido no orifício 12 , ou seja $20 \%$ do crescimento encontrado no orifício do controle positivo. A CIM 50\% foi calculada de maneira semelhante.

O ponto de corte para determinar isolamentos sensiveis e resistentes foi aquele proposto no documento M27 A, $\operatorname{NCCLS}{ }^{(11)}$, considerando a amostra sensível ao fluconazol se CIM $\leq 8 \mu \mathrm{g} / \mathrm{ml}$; com sensibilidade dependente da dose (SDD) se CIM $=16 \mu \mathrm{g} / \mathrm{ml}$ ou $32 \mu \mathrm{g} / \mathrm{ml}$; e resistente se $\mathrm{CIM} \geq$ $64 \mu \mathrm{g} / \mathrm{ml}$. Para itraconazol, as amostras foram consideradas sensíveis quando $\mathrm{CIM} \leq 0,125 \mu \mathrm{g} / \mathrm{mL}$; com $\mathrm{SDD}$ se $\mathrm{CIM}=$ $0,25 \mu \mathrm{g} / \mathrm{mL}$ ou $0,5 \mu \mathrm{g} / \mathrm{mL}$; e resistente se $\mathrm{CIM} \geq 1 \mu \mathrm{g} / \mathrm{mL}$. Para anfotericina $\mathrm{B}$, considerou-se sensível a amostra cujo resultado de $\mathrm{CIM}$ foi $\leq 1$.

\section{Resultados}

Entre as 250 pacientes participantes da pesquisa, $86 \%$ estiveram na faixa etária variando de 16 a 45 anos.

Dos 250 esfregaços de fluido vaginal submetidos à coloração de Gram, 42 (16,8\%) e 69 (27,6\%) entre as culturas foram positivas para leveduras.

A levedura isolada mais freqüente foi Candida albicans (74\%), seguida de Candida glabrata em 14,5\% das amostras. As outras espécies, Candida tropicalis e Candida parapsilosis, totalizaram oito $(11,5 \%)$ culturas positivas (Tabela 1).
Resultados da identificação das leveduras isoladas nas 69 amostras Tabela 1 de fluido vaginal positivo na cultura
Leveduras isoladas

Candida albicans

Candida glabrata

Candida tropicalis

Candida parapsilosis

Total
Amostras de fluido vaginal Total

$\%$

$51 \quad 74$

$10 \quad 14,5$

$5 \quad 7,2$

34,3

69
A CIM foi determinada frente a 68 leveduras isoladas, $\mathrm{e}$ os resultados foram avaliados para $50 \%\left(\mathrm{IC}_{50}\right)$ e $80 \%\left(\mathrm{IC}_{80}\right)$ de inibição.

Os 51 isolados de Candida albicans apresentaram os valores de $\mathrm{CIM}-\mathrm{IC}_{50}$ variando de 0,12 a $16 \mu \mathrm{g} / \mathrm{ml}$ para fluconazol e de 0,03 a $8 \mu \mathrm{g} / \mathrm{ml}$ para itraconazol; e de $\mathrm{IC}_{80}$, de 0,25 a $64 \mu \mathrm{g} / \mathrm{ml}$ para fluconazol e de 0,03 a $16 \mu \mathrm{g} / \mathrm{ml}$ para itraconazol (Tabela 2). No caso de fluconazol, $94,1 \%$ dos isolados tinham valores de $\mathrm{CIM}^{-\mathrm{IC}} \mathrm{C}_{50}$ entre 0,12 e $8 \mu \mathrm{g} / \mathrm{ml}$ e $5,9 \%$, de $16 \mu \mathrm{g} / \mathrm{ml}$, correspondendo ao valor de sensibilidade dependente da dose. No caso de leitura com $\mathrm{IC}_{80}$ observou-se aumento da CIM em 9,8\% $(n=5)$ dos isolados, que passaram a ter valor correspondente a resistente (>32 $\mu \mathrm{g} / \mathrm{ml}$ ) (Figura 1). Em relação ao itraconazol, quando se considerou a $\mathrm{IC}_{50}, 98 \%$ das amostras foram sensíveis, e apenas um isolado apresentou CIM de $8 \mu \mathrm{g} / \mathrm{ml}$. Com leitura de $\mathrm{IC}_{80}, 70,6 \%$ foram sensíveis; $11,8 \%$, sensíveis dependendo da dose; e 17,6\%, resistentes (Figura 2). Em relação à anfotericina $\mathrm{B}$, os valores de CIM variaram entre 0,12 e $1 \mu \mathrm{g} / \mathrm{ml}$, sendo todas consideradas sensíveis pelos parâmetros atuais (Tabela 2, Figura 3).

Os isolados de espécies não-albicans apresentaram valores de CIM para fluconazol entre 0,25 e $64 \mu \mathrm{g} / \mathrm{ml}$ para $\mathrm{IC}_{50^{\prime}} 0,5$ e $64 \mu \mathrm{g} / \mathrm{mL}$ para IC $\mathrm{I}_{80^{\prime}}$ e $11,7 \%$ dos isolados apresentaram valor de $64 \mu \mathrm{g} / \mathrm{ml}$, considerado resistente, mesmo realizando-se cálculo em IC ${ }_{50}$. Quando se considerou o IC ${ }_{80}, 23,5 \%$ dos isolados foram sensível-dependentes da dose, e igual valor apresentou resistência (Figura 4). No caso de itraconazol, os valores das CIMs variaram entre 0,03 e $16 \mu \mathrm{g} / \mathrm{ml}$ tanto para $\mathrm{IC}_{50}$ como para IC $\mathrm{IC}_{80}$. Os valores de CIM entre 0,25 e $0,5 \mu \mathrm{g} / \mathrm{ml}$, considerados sensíveis dependendo da dose, foram observados em 41,2\% $(n=7)$ dos isolados, e 23,5\% $(n=4)$ apresentaram resistência. Quando a IC foi calculada para $80 \%$ de inibição, $11,8 \%$ dos isolados apresentaram valores sensível-dependentes da dose e 70,6\%, resistentes (Figura 5). Os resultados de CIM 


\section{Variações das concentrações inibitórias minimas para fluconazol, itraconazol e anfotericina}

$B$ de isolados de Candida albicans do fluido vaginal de 51 pacientes, em relação à leitura com

Tabela $250 \%\left(\mathrm{IC}_{50}\right), 80 \%\left(\mathrm{IC}_{80}\right)$ e $100 \%\left(\mathrm{IC}_{100}\right)$ de inibição

\begin{tabular}{|c|c|c|c|}
\hline \multirow[t]{2}{*}{ Droga } & \multicolumn{3}{|c|}{ Variações das CIMs $(\mu \mathrm{g} / \mathrm{ml})$} \\
\hline & $\mathrm{IC}_{50}{ }^{*}$ & $\mathrm{IC}_{80}$ ** & $\mathrm{IC}_{100}^{* * *}$ \\
\hline Fluconazol & $\leq 0,12-16$ & $0,25-64$ & \\
\hline Itraconazol & $\leq 0,03-8$ & $\leq 0,03-16$ & \\
\hline Anfotericina B & & & $0,12-1$ \\
\hline
\end{tabular}
mínima $-100 \%$ de inibição do crescimento.

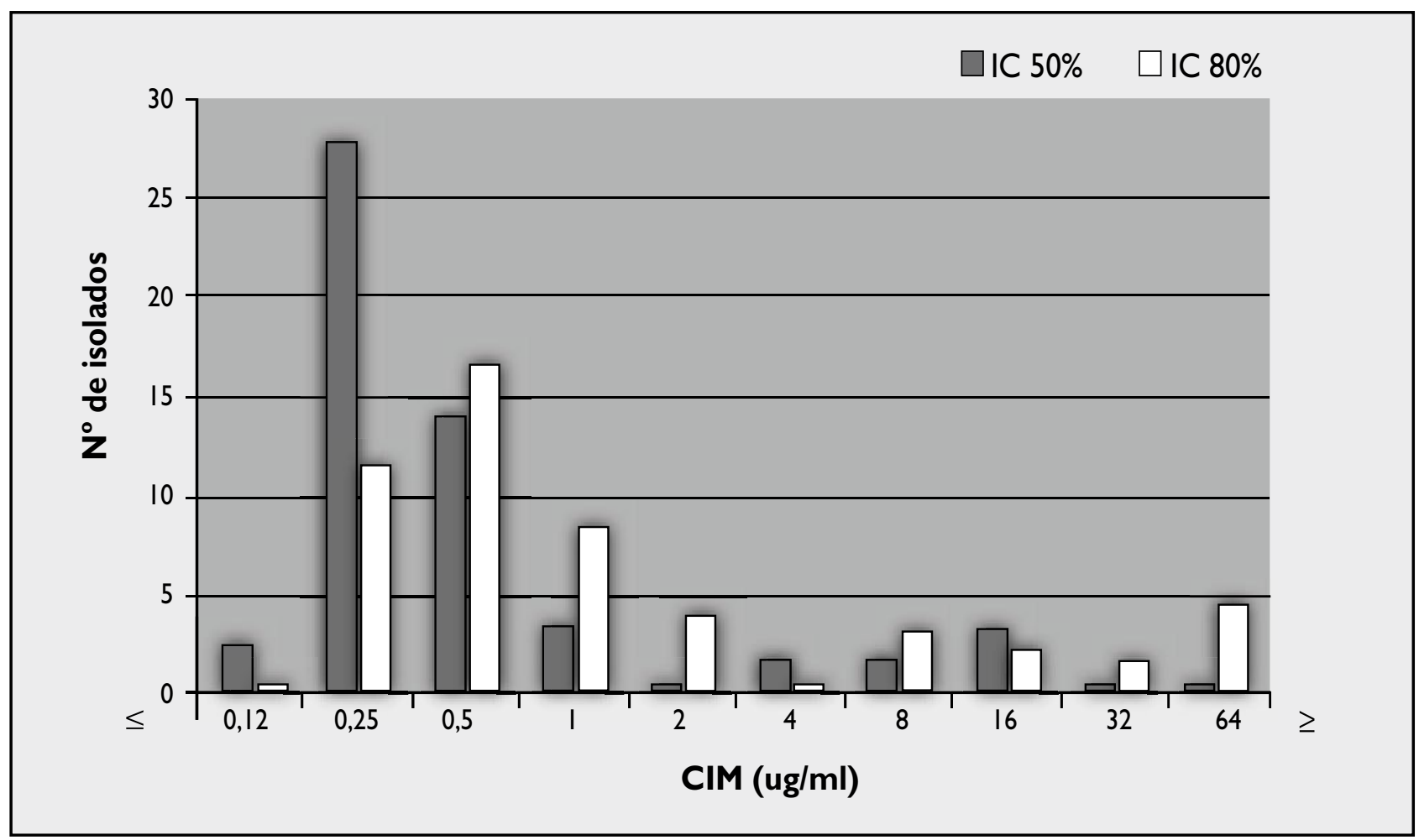

Figura 1 - Distribuição dos valores de concentração inibitória mínima (CIM) de fluconazol, calculados com índice de inibição de 50\% e 80\%, em isolados de Candida Albicans

para anfotericina B variaram entre 0,12 e $2 \mu \mathrm{g} / \mathrm{ml}$, sendo que apenas um isolado apresentou CIM de $2 \mu \mathrm{g} / \mathrm{ml}$ (Tabela 3, Figura 6).

\section{Discussão}

Candida albicans continua a ser o agente mais prevalente de candidíase vulvovaginal, embora nos últimos anos as espécies denominadas não-albicans tenham se tornado também importantes, principalmente em face dos casos de resistência apresentados por algumas destas $(1,3,5,17)$.
Pizzolito e Mendes-Giannini ${ }^{(13)}$ isolaram de 62 fluidos vaginais de 29 mulheres com CVV: Candida albicans em $87 \%$, seguida de Candida famata (5,5\%), Candida glabrata (3,7\%) e Candida guilliermondii (3,7\%).

Linhares et al. ${ }^{(10)}$ verificaram também que C.albicans foi a levedura mais isolada, seguida por C. glabrata, C.parapsilosis e C. tropicalis em casos de CVV. Otero et al.(12) verificaram que Candida albicans (89,3\%) foi a levedura mais isolada de casos de CVV em profissionais do sexo na Espanha.

$\mathrm{Xu}$ et al. ${ }^{(20)}$ estudaram leveduras do sistema genitourinário em três grupos de mulheres sem sintomatologia 


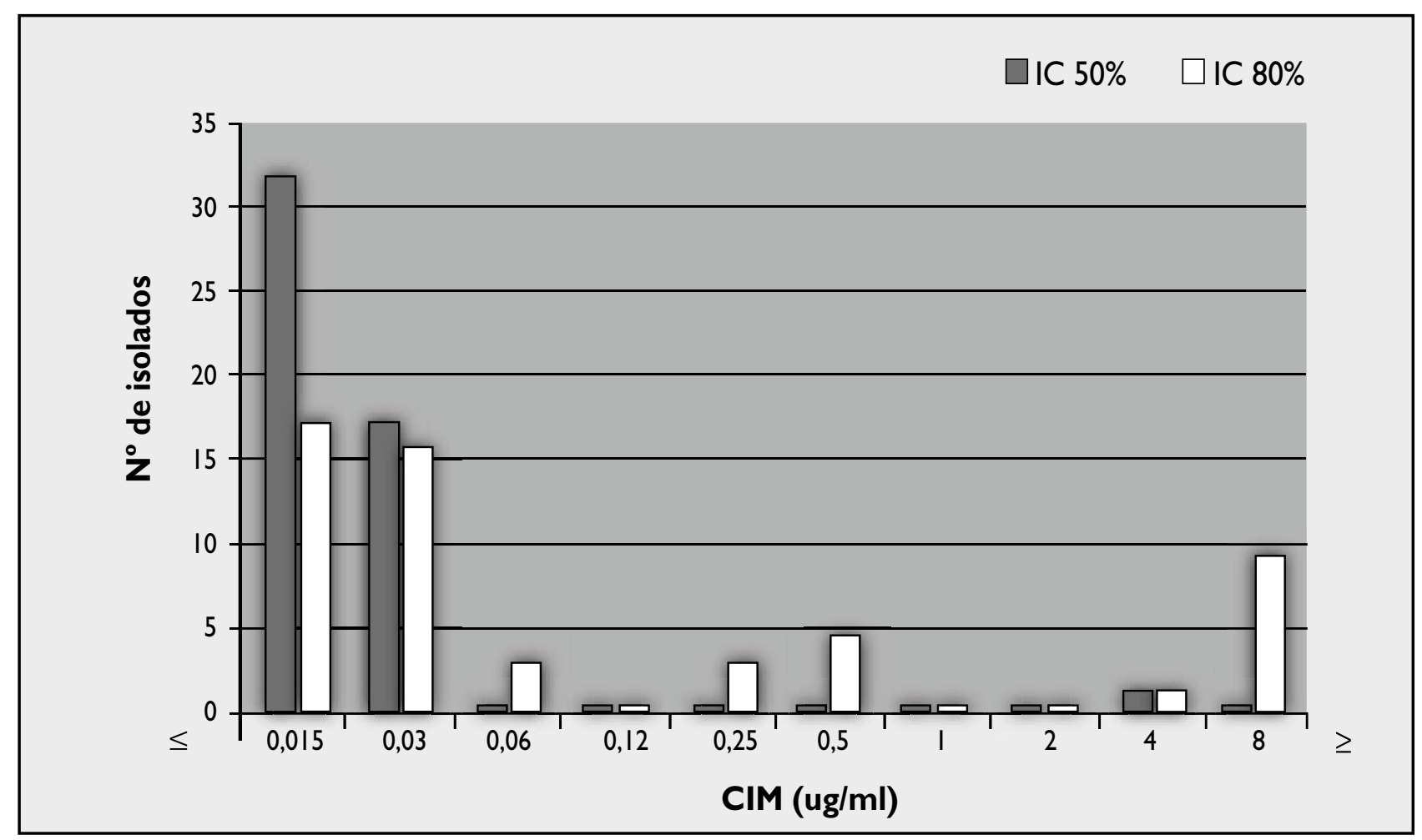

Figura 2 - Distribuição dos valores de CIM de itraconazol, calculados com índice de inibição de 50\% e 80\%, em isolados de Candida albicans do fluido vaginal de 51 pacientes

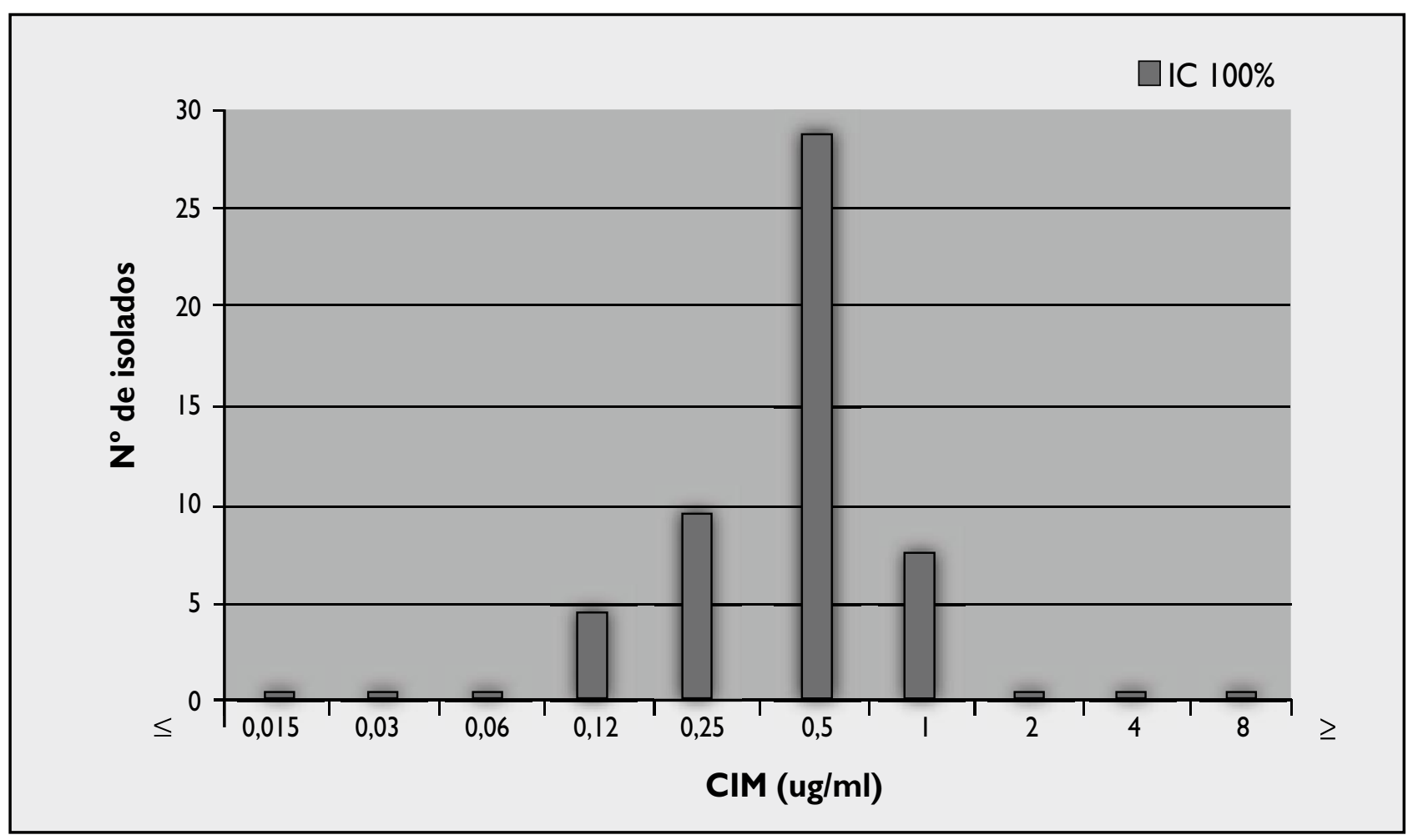

Figura 3 - Distribuição dos valores de CIM de anfotericina B, calculados para índice de inibição de 100\%, em isolados de Candida albicans do fluido vaginal de 51 pacientes 


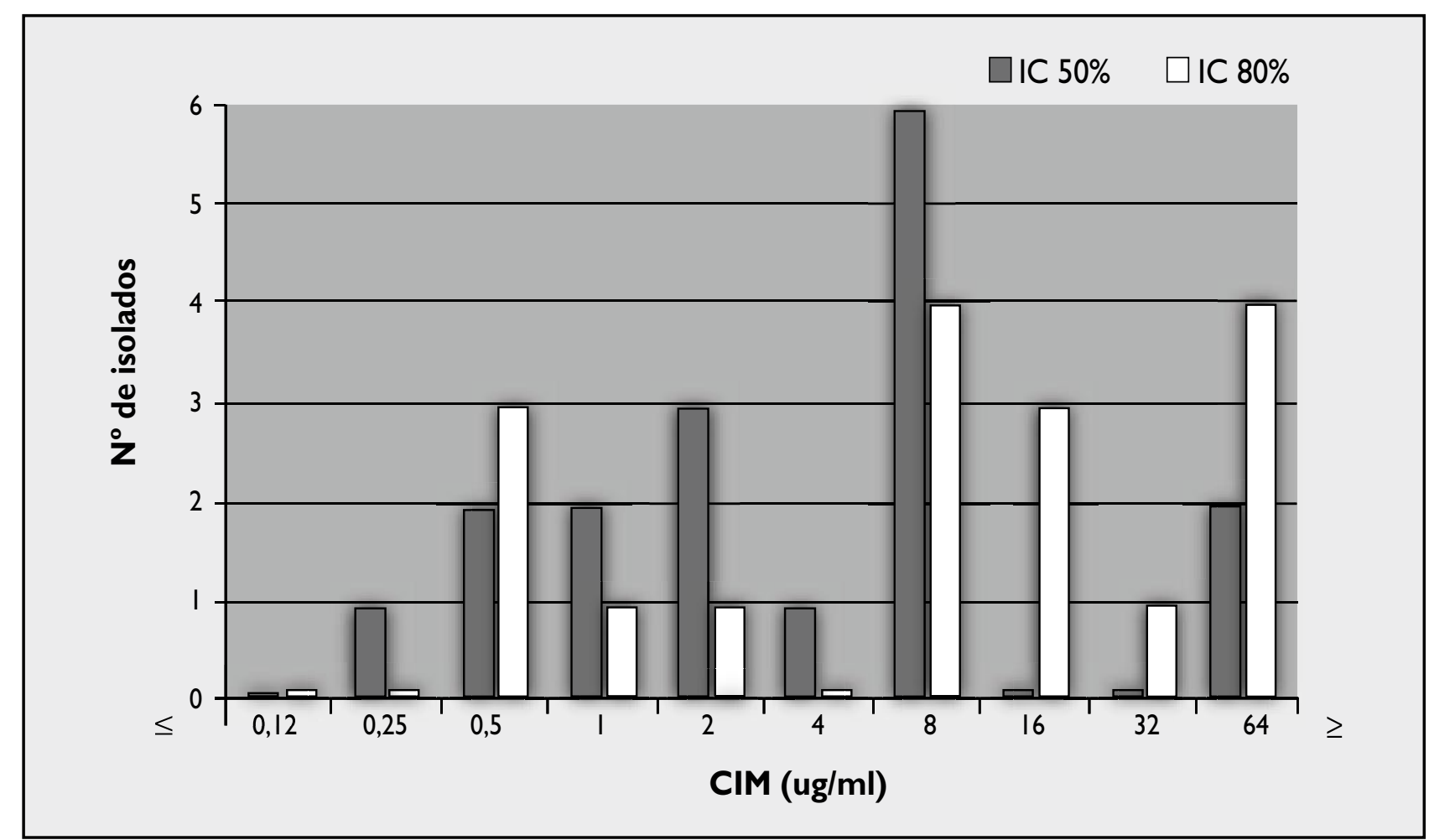

Figura 4 - Distribuição dos valores de CIM de fluconazol, calculados para índice de inibição de 50\% e 80\%, em isolados de espécie não-albicans do fluido vaginal de 17 pacientes

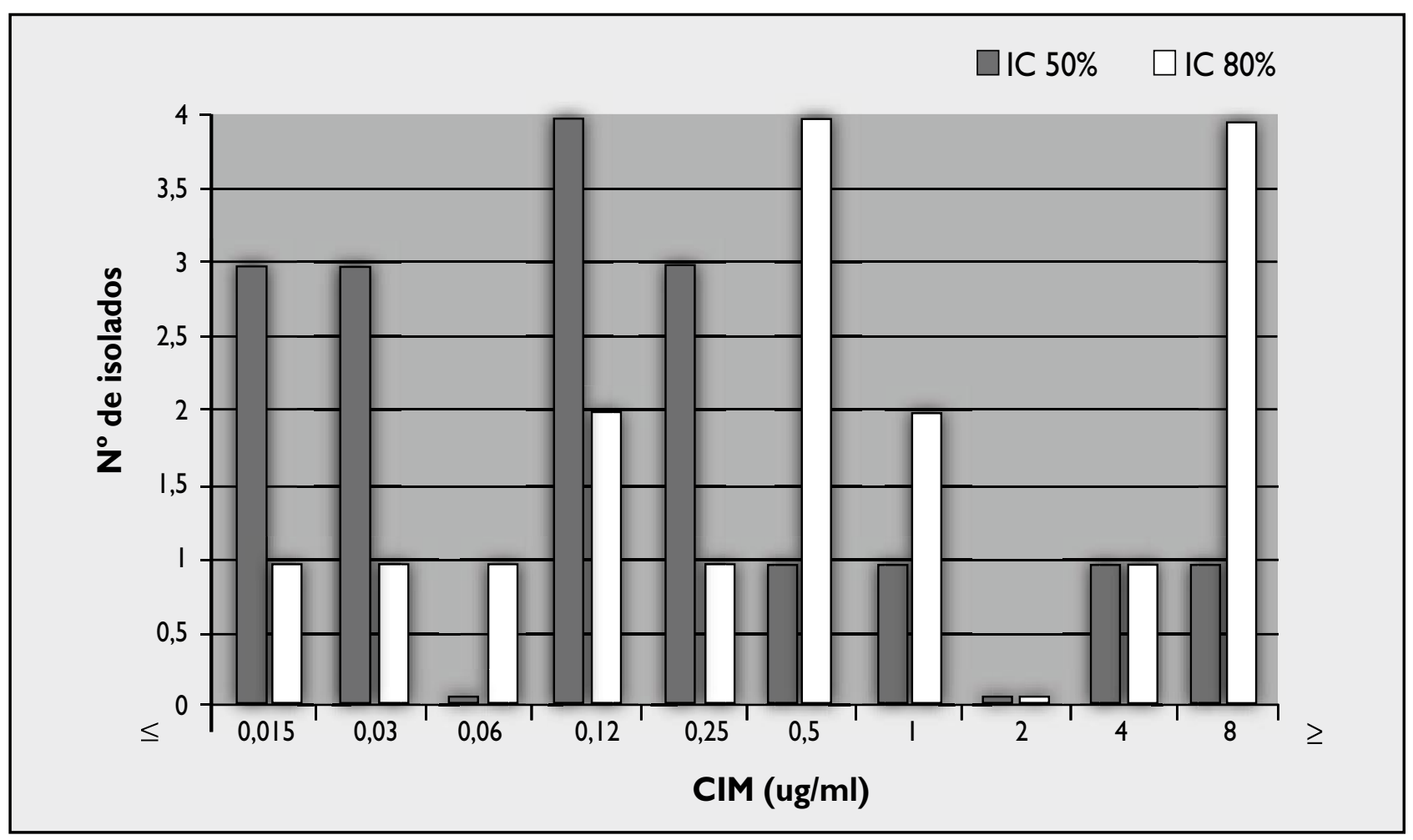

Figura 5 - Distribuição dos valores de CIM de itraconazol, calculados para índice de inibição de 50\% e 80\%, em isolados de espécies não-albicans do fluido vaginal de 17 pacientes 
Variaçōes das concentraçōes inibitórias minimas para fluconazol, itraconazol e anfotericina

$B$ de isolados do gênero Candida espécie não-albicans do fluido vaginal de 17 pacientes, em

Tabela 3 relação à leitura com 50\% (IC $\left.{ }_{50}\right), 80 \%\left(\mathrm{IC}_{80}\right)$ e $100 \%\left(\mathrm{IC}_{100}\right)$ de inibição

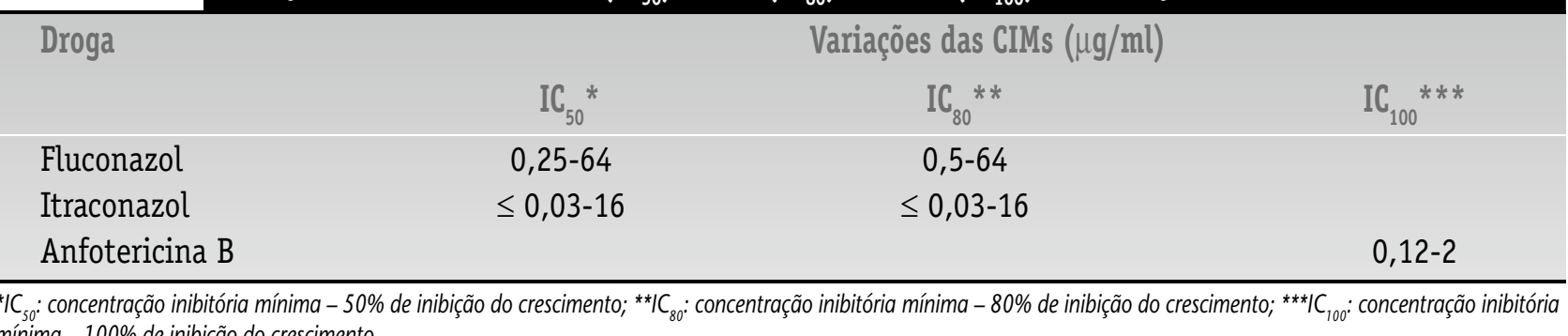
mínima - 100\% de inibição do crescimento.

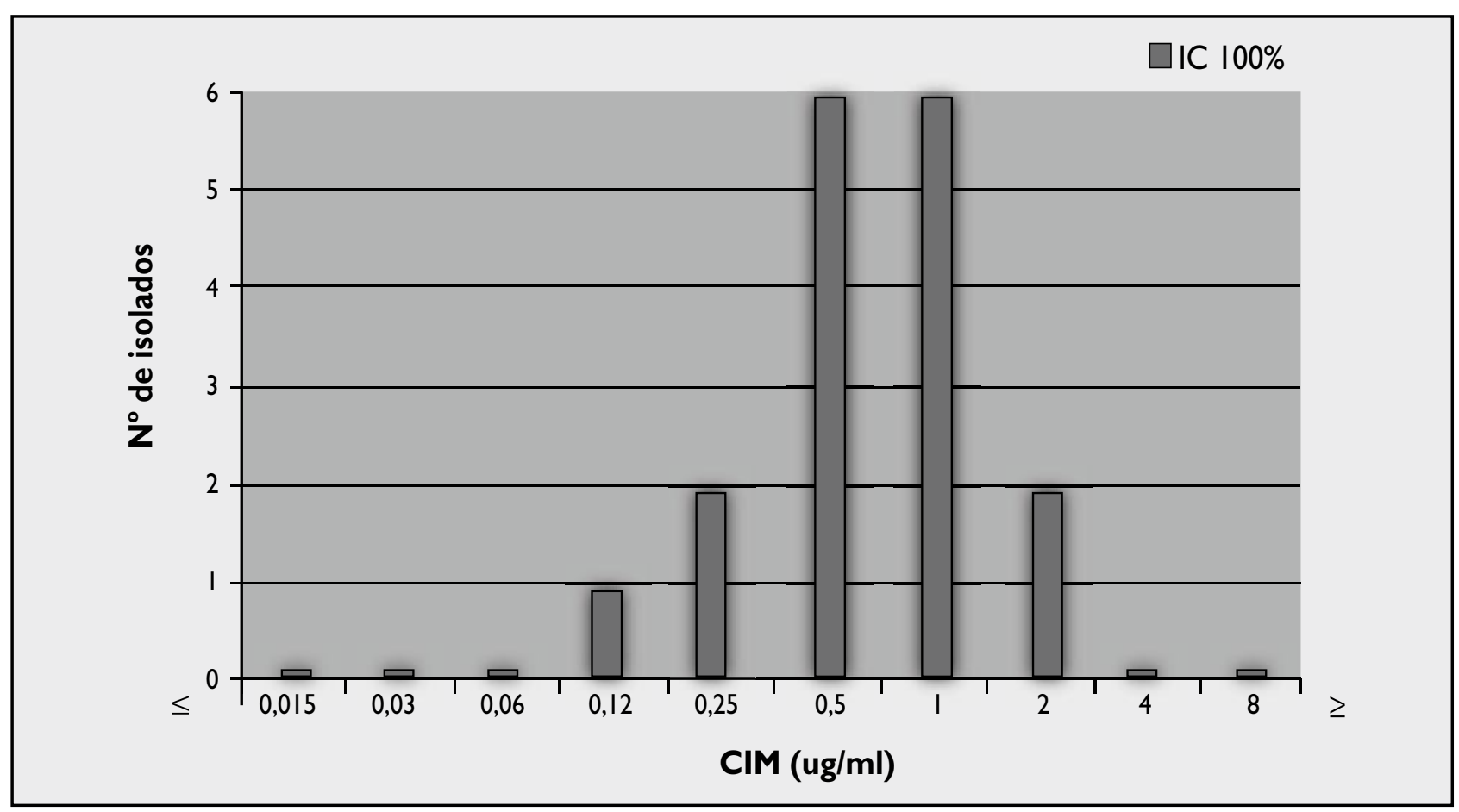

Figura 6 - Distribuição dos valores de CIM de anfotericina B, calculados para índice de inibição de 100\%, em isolados de espécies não-albicans do fluido vaginal de 17 pacientes

aparente para CVV e verificaram que C. albicans foi a mais prevalente.

Nesta pesquisa, leveduras foram isoladas em $27 \%$ das pacientes, independente da presença de sintomas para CVV. Candida albicans foi a levedura mais prevalente (74\%), seguida de C. glabrata (14,5\%), Candida tropicalis $(7,3 \%)$ e Candida parapsilosis (4,2\%).

Os resultados deste trabalho são concordantes com os resultados das experiências dos autores acima, em que C. albicans continua a ser a levedura mais isolada de CVV, porém, especial atenção deve ser dispensada à presença de C. glabrata em porcentuais maiores que o observado em pesquisas, tanto no Brasil como no exterior. C. albicans ainda é responsável por mais de $90 \%$ dos casos de CVV, mas, na última década, um aumento de casos de vaginites por espécies não-albicans foi descrito, e, entre elas, C. glabrata foi a mais comum $(4,15,18)$.

O aumento desta levedura, em casos de CVV, pode ser reflexo da realização de culturas e provas de identificação, porém, não se pode descartar a possibilidade de este aumento acontecer em razão do uso de terapias seletivas com doses inadequadas, tanto tópicas quanto sistêmicas, com antimicóticos.

Vários antifúngicos são empregados no tratamento de $\mathrm{CVV}$, e as drogas azólicas se constituem no tratamento de eleição desta doença. No entanto, $5 \%$ a $25 \%$ das pacientes apresentam recidivas que podem redundar em alguns casos em CVV crônica. O uso de fluconazol de forma profilática 
em doses baixas para prevenir infecções fúngicas em pacientes imunocomprometidas tem levado à seleção de leveduras da microbiota, fazendo com que apareçam as espécies não-albicans, como C. grablata e C. krusei, ambas resistentes a este antifúngico(15).

Neste trabalho, verificou-se que todos os isolados de C. albicans foram sensiveis a anfotericina B, mas 5,9\% apresentaram SDD a fluconazol e $2 \%$ de resistência ao itraconazol.

No caso de isolados não-albicans, $11,7 \%$ e 23,5\% foram resistentes para fluconazol e itraconazol, respectivamente, e um isolado de C. glabrata apresentou CIM de $2 \mu \mathrm{g} / \mathrm{ml}$ para anfotericina $B$. Testes de sensibilidade aos antifúngicos não são solicitados rotineiramente, no entanto, em função do aparecimento de porcentual bastante significativo de leveduras com SDD ou resistentes, sugere-se um programa para o desenvolvimento de testes de sensibilidade para o monitoramento de amostras vaginais, procurando correlacionar estes achados com os achados in vivo.

\section{Agradecimentos}

Agradecemos à Dra. Márcia de Souza Carvalho MeIhem, a Mônica M. Peria (Instituto Adolfo Lutz/Laboratório Central) e aos funcionários do Laboratório de Micologia da UNESP/campus de Araraquara o apoio técnico.

\section{Referências}

I. ACOG TECHNICAL BULLETIN. Committee on Technical Bulletins of the American College of Obstetricians and Gynecologists. Vaginitis. Int J Gynaecol Obstet, v. 54 , p. 293-302, 1996.

2. BAUTERS, T. G.; DHONT, M. A.;TEMMERMAN, M. l.; NELIS, H. J. Prevalence of vulvovaginal candidiasis and susceptibility to fluconazole in women. Am J Obstet. Gynecol, v. 3, p. 568-74, 2002.

3. BRASIL. Ministério da Saúde. Manual de Controle das Doenças Sexualmennte Transmissíveis: DST. 3.ed. Brasília, DF, 1999. p. 77-9.

4. CAUWENBERGH, G. Vaginal candidiasis: envolving trends in the incidence and treatment of non-Candida infections. Curr Probl Obstet Gynecol Fertil, v. 8, p. 24I-5, 1990.

5. ECKERT, L. O.; HAWES, S. E.;STEVES, C. E.Vulvovaginal candidiasis: clinical manifestations, risk factors, management algorithm. Obstet Ginecol, v. 92, p. 757-65, 1998.

6. EYLER, A. E.; PIERSON, C.; REED, B. D. Improved diagnosis of candida vulvovaginitis using Diamond's media modified. J Women's Health,v. 5, p. 79-84, 1996.

7. FOSTER, D. C.Vulvitis and vaginitis. Curr Opinion Obstet Gynecol, v. 5, p. 726-32, 1993.

8. KWON-CHUNG, K. J.; BENNETT, M. D. Medical mycology. Philadelphia: Lea \& Febiger, 1992. p. 280-335.

9. LACAZ, C. S.; POTO, E.; MARTINS, J. E. C.; HENINS-VACCARI, E. M.; MELO, N.T. Tratado de Micologia Médica Lacaz. 9. ed. São Paulo: Sarvier, 2002. p. 128.

I0. LINHARES, L. M.; WITKIN, S. S.; MIRANDA, S. D.; FONSECA A. M.; PINOTTI, J.A.; LEDGER,W. J. Differentiation between women with vulvovaginal syntoms who are positive or negative for Candida species by culture. Infect Dis Obstet Gynecol, v. 4, p. 22 I-5, 200 I.

II. NATIONAL COMMITTEE FOR CLINICAL LABORATORY STANDARDS, 1997. Reference method for broth dilution antifungal susceptibility testing of yeasts. Approved standard M27-A. National Committee for Clinical Laboratory Standards, Wayne, PA.
12. OTERO, L.; PALACIO,V.; CARRENO, F;; MENDEZ, F. J:VAZQUEZ, F. Vulvovaginal candidiasis in female sex workers. Int J STD AIDS, v. 9, p. 526-30, 1998.

13. PIZZOLITTO, E. L.; MENDES-GIANNINI, M. J. S. Candidíase vulvovaginal com episódios de repetição: epidemiologia e biotipagem de isolados de Candida albicans. Rev Ciênc Farm, São Paulo, v. 22, p. |47-63, 2001.

14. RODRÍGUEZ-TUDELLA, J. L.; RODERO, L.; CUENCAESTRELA, M.; CÓRDOBA, S. III Curso Hispano-Argentino de Micologia Médica, Buenos Aires, Argentina. Determinacion de la resistencia a los antifúngicos en el laboratorio, 2001. Manual digitado.

I5. SAPORITI, A. M.; GÓMEZ, S. L.; GALEANO, M.; DAVEL, G.; VOVOT, W:; RODERO, L. Candidiasis vaginal: etiologia y perfil de sensibilidade a agentes antifúngicos de uso clínico. Rev Argent Microbiol, v. 33, p. 217-22, 2001.

16. SOBEL, J. D. CandidalVulvovaginitis. Clin Obstet Gynecol, Detroit, v. 36, n. I, p. 153-65, mar. 1993.

17. SOBEL, J. D.Vaginitis. N Engl J Med, v. 337, p. I 896-903, dec. 1997.

I 8. SOBEL, J. D.; CHAIN,W.Treatment of Torulopsis glabrata vaginitis: reptrospective review of boric acid therapy. Clin Infect Dis, v. 24, p. 649-52, 1997.

19. STEBEN, M. Sexually transmitted diseases. Disponível em: <http://sogc.medical.org/sogc_docs/public/guidelines/sextrl. htm>. Acesso em: 12 ago. 2000

20. XU, J.; BOYD, C. M.; LIVINGSTON, E.; MEYER, W.; MADDEN, J. F.; MITCHELL, T. G. Species and genotypic diversities and smilarities of pathogenic yeasts colonizing women. J Clin Microbiol, v. 37, p.3835-43, 1999.

\begin{tabular}{l|l} 
& \\
& Endereço para correspondência \\
\hline & Leonilda Chiari Calle \\
& Instituto Adolfo Lutz - Laboratório Regional de \\
Presidente Prudente & \\
Av. Coronel José Soares Marcondes 2.357 \\
CEP 19013-050 - Presidente Prudente-SP \\
Tels.: (18) 221-1449/221-1888 \\
Fax: (18) 221-5814 \\
e-mail: leonilda@ial.sp.gov.br
\end{tabular}

